\title{
COMPETENCIA EMOCIONAL Y RENDIMIENTO ACADÉMICO EN EL ALUMNADO UNIVERSITARIO
}

Iratxe Suberviola-Ovejas1: Universidad de la Rioja. España iratxe.suberviola@unirioja.es

\section{RESUMEN}

Este estudio presenta la finalidad de evidenciar los beneficios de la inclusión de la Educación Emocional en las diferentes etapas educativas, concretamente como fomento del rendimiento académico del alumnado universitario. Para ello, se realiza un análisis en el que se correlacionan la variable puntuaciones obtenidas en el TMMS24 con la variable calificaciones académicas en una muestra de alumnos y alumnas de $1^{\circ}$ curso de grado de la Universidad de La Rioja. Los instrumentos utilizados son: el TMMS-24; El examen teórico; Rúbrica para las prácticas. Se lleva a cabo un análisis cuantitativo correlacional, entre la variable puntuaciones obtenidas en el TMMS-24 y la variable calificaciones académicas, a tres niveles; 1) de toda la muestra y dividida por los grupos establecidos según las puntuaciones obtenidas en el TMMS-24 en cada una de las sub-escalas; 2) la comparación de los promedios en las calificaciones teórica y práctica, según los cuartiles establecidos con las puntuaciones obtenidas en el TMMS-24; 3) el análisis correlacional de las variables, aplicando la prueba de Pearson emparejando, tanto la puntuación total como la puntuación de las tres subescalas del TMMS-24, con las calificaciones obtenidas en la parte práctica y la teórica.

PALABRAS CLAVE: Educación emocional - Competencias emocionales - Inteligencia emocional - Rendimiento académico

\footnotetext{
${ }^{1}$ Autor correspondiente Iratxe Suberviola-Ovejas: Contratada interina, Departamento Ciencias del Educación. Universidad de la Rioja. (España).

Correo: iratxe.suberviola@unirioja.es
} 


\title{
EMOTIONAL COMPETENCE AND ACADEMIC PERFORMANCE IN UNIVERSITY STUDENTS
}

\begin{abstract}
This study presents the purpose to demonstrate the benefits in the incorporation of the Emotional Education in the different educational stages, concretely as a promotion of the academic performance of the university students. For it, we realized an analysis in which there correlate the variable punctuations obtained in the TMMS-24 with the variable academic qualifications in a sample of students of $1^{\circ}$ degree in the University of La Rioja. The used instruments are: the TMMS-24; the theoretical exam; Paragraph for the practices. A correlational quantitative analysis is realized, between the variable punctuations obtained in the TMMS-24 and the variable academic qualifications, to three levels; 1) Of the whole sample and divided by the groups established according to the punctuations obtained in the TMMS-24 in each of the sub-scales; 2) the comparison of the averages in the qualifications theoretical and practical, according to the quartiles established with the punctuations obtained in the TMMS-24; 3) the analysis co relational of the variables, applying Pearson's test, pairing both the total punctuation and the punctuation of three subscales of the TMMS-24, with the qualifications obtained in the practical part and the theoretical one.
\end{abstract}

KEY WORDS: Emotional education - Emotional competence - Emotional intelligence - Academic Performance

\section{INTRODUCCIÓN}

Desde su aparición, el término Inteligencia Emocional (IE) ha sido un constructo con una repercusión importante que ha generado un amplio número de investigaciones y publicaciones al respecto, modificando "obsoletas teorías" sobre el concepto de inteligencia. En la escuela tradicional se consideraba que un niño era inteligente cuando dominaba ciertas materias como las lenguas clásicas, las matemáticas, etc. Posteriormente, con la introducción del término Cociente Intelectual (CI), para catalogar a un alumno como inteligente, éste debía tener una cierta puntuación en los test de inteligencia. Este argumento se sustentaba en la relación existente entre el CI de los alumnos y su rendimiento académico, de modo que, el alumnado con mayores puntuaciones en los test de inteligencia obtenían mejores resultados académicos.

Actualmente sin embargo, autores como Fernández-Berrocal y Extremera (FernándezBerrocal y Extremera, 2003) postulan que esta concepción ha entrado en crisis debido a 
a) La inteligencia académica no es suficiente para alcanzar el éxito profesional, puesto que los profesionales más prestigiosos son los que tienen la habilidad de conocer y manejar sus emociones y las de los otros de un modo eficaz.

b) La inteligencia en términos de CI no garantiza el éxito en nuestra vida cotidiana, no contribuye a nuestro equilibrio emocional ni a nuestra salud mental.

En este sentido, una de las líneas de investigación que mayor interés ha generado en los últimos años en el ámbito de la IE ha sido el papel que ésta juega en el contexto educativo y, especialmente en la influencia que tiene como determinante del éxito académico y la adaptación social de los estudiantes. Como anteriormente se ha apuntado, tradicionalmente se otorgaba el protagonismo principal del éxito al desarrollo y aplicación de las funciones meramente cognoscitivas. Sin embargo, durante estas dos últimas décadas se han llevado a cabo diversos estudios sobre el papel que cumplen las habilidades emocionales en el aprendizaje. A este hecho ha contribuido el reconocimiento por parte de los profesionales del ámbito educativo del impacto generalizado que las emociones tienen en todos los aspectos de la vida y, específicamente en el rendimiento de los individuos. (Fernández-Berrocal y Extremera, 2006).

\subsection{Evaluación de las competencias emocionales}

Una de las líneas de estudio con mayor peso empírico en cuanto a número de investigaciones desarrolladas es la que pretende la medición y evaluación de la Inteligencia Emocional y de las competencias que la forman. Los diferentes métodos de valoración que nos aporta la literatura pueden ser clasificados en tres modalidades, pudiéndose observar en cada una de ellas luces y sombras:

\subsection{Cuestionarios, escalas y autoinformes}

Generalmente estos instrumentos están formados por enunciados verbales cortos en los que el sujeto evalúa su nivel de inteligencia emocional a través de una escala Likert. Así pues, la persona da una valoración subjetiva de sus niveles en ciertas habilidades y competencias afectivas. Este método evaluativo es uno de los más frecuentes. En cuanto a las medidas de autoinforme más conocidas dentro de la IE, cabe destacar el Trait Meta-Mood Scale (TMMS) de Salovey, Mayer, Goldman, Turvey y Palfai (1995) y su versión reducida y adaptada al castellano, el TMMS-24 de Fernández-Berrocal, y colaboradores (Fernández-Berrocal, Extremera y Ramos, 1999). El objetivo de ambas es obtener una estimación personal sobre los aspectos reflexivos de la experiencia emocional. La primera consiste en una escala de rasgo de metaconocimiento de los estados emocionales que evalúa a través de 48 items. Consta de tres factores: 1) Atención a las emociones v capacidad para identificarlas 
Claridad emocional como la capacidad para comprenderlas en uno mismo y en los demás y 3) Reparación emocional como la capacidad para manejar las emociones e influir en nuestros pensamientos. A la persona se le pide que evalúe el grado en el que está de acuerdo con cada uno de los ítems sobre una escala de tipo Likert de 5 puntos. (1=totalmente en desacuerdo, 5=totalmente de acuerdo) El segundo instrumento presenta las mismas características y categorías que la primera con la diferencia del número de ítems que se reduce a la mitad.

\subsection{Observadores externos}

Esta forma de evaluación parte del presupuesto de que si la IE implica la capacidad para manejar y comprender nuestras propias emociones y las de las personas que nos rodean, ¿por qué no preguntar a las personas próximas a nosotros sobre cómo manejamos nuestras emociones en público y la forma en que afrontamos los problemas y sucesos que nos suceden? (Extremera y Fernández-Berrocal, 2004a).

$\mathrm{Su}$ objetivo consiste en obtener información, no únicamente a través del individuo evaluado, sino también de aquellos que comparten su tiempo, puesto que resultan fuentes de información valiosa a la hora de analizar las competencias socioemocionales de cada individuo y poder analizar los puntos fuertes y las debilidades que deben entrenarse. Algunos de los más conocidas son: ECI-360 (Boyatzis y Burckel, 1999); TEIQue-360SF (Petrides y Furnham, 2011); EQ-360 (Bar-On y Handley, 1997).

\subsection{Tareas emocionales o de habilidad}

Este tipo de evaluación también se denomina como medidas de ejecución. Surge para suplir los posibles sesgos que presentan los métodos basados en cuestionarios, escalas o autoinformes y los métodos basados en observadores externos. El objetivo de estos instrumentos es, por un lado evitar la falsificación de las respuestas por los propios sujetos en aquellas situaciones en las que se desea dar una imagen positiva y, por otro, disminuir los sesgos perceptivos y situacionales provocados por los observadores externos. (Extremera y Fernández-Berrocal, 2004a) La fundamentación de estas herramientas es que para evaluar si una persona es hábil o no en un ámbito concreto, en este caso el emocional y afectivo, la mejor forma de hacerlo es comprobar sus habilidades a través de diferentes ejercicios que requieren poner a prueba tales competencias en comparación con unos criterios de puntuación predeterminados y objetivos (Mayer, Caruso y Salovey, 1999). Algunos de los instrumentos que podemos encontrar dentro de este tipo de evaluación son: MSCEIT (Mayer, Salovey y Caruso, 2002); ACES (Trentacosta y Izard, 2007). 


\subsection{Relación entre las competencias emocionales y variables socio-educativas}

Una de las principales motivación por evaluar las competencias emocionales es poder realizar correlaciones con otras variables que inciden en el alumnado como: relaciones sociales, conductas disruptivas, rendimiento académico, equilibrio personal, consumo de sustancias y comportamientos delictivos, etc.

\subsection{Inteligencia emocional y equilibrio personal}

Los seres humanos somos la unión entre lo cognitivo, lo afectivo, lo social, lo cultural, etc. En este sentido el bienestar psicológico y, en especial el de los adolescentes, influye en su calidad de vida, incidiendo a su vez en un sentido positivo o negativo en sus relaciones sociales. Existe una clara relación entre bienestar psicológico, equilibrio emocional y ciertos aspectos educativos. Este hecho se muestra en un estudio realizado por Salovey, Stroud, Woolen y Epel (2002) con estudiantes universitarios que revela como aquellos que presentan mayor IE percibida a través del (TMMS) muestran menores síntomas físicos, menor ansiedad social o depresión, una elevada autoestima unida a una mayor satisfacción interpersonal y un mejor empleo de estrategias de afrontamientos en situaciones problemáticas.

En España un grupo de investigadores (Fernández-Berrocal, Alcaide y Ramos 1999) realizaron una investigación con alumnado adolescente de secundaria donde los resultados mostraban que cuando a los jóvenes se les divide en grupos en función de su sintomatología, los que no presentaban síntomas de depresión se diferenciaban de los que sí los mostraban en niveles superiores de IE, concretamente, por una mayor claridad hacia sus sentimientos y una elevada competencia en reparación de sus emociones. En cambio, los estudiantes depresivos presentaban mayores puntuaciones de ansiedad y pensamientos rumiantes. Igualmente altas puntuaciones en IE se han asociado a puntuaciones más elevadas en autoestima, felicidad, salud mental y satisfacción vital y, menores puntuaciones en ansiedad, depresión y supresión de pensamientos negativos. (Fernández-Berrocal, Alcaide, Extremera y Pizarro, 2006).

\subsection{Inteligencia emocional y relaciones sociales}

Las personas emocionalmente competentes tienen la capacidad de percibir, comprender y manejar sus propias emociones pero también presentan habilidades de percepción, comprensión y manejo de las emociones de los demás.

Esta teoría proporciona un nuevo marco para la investigación de la adaptación social y escolar, puesto que la IE juega un papel elemental en el establecimiento, mantenimiento y calidad de las relaciones interpersonales. No se debe obviar la importancia que estas relaciones juegan en el ámbito escolar, tanto con los iguales, 
Con respecto a esta vinculación, encontramos diversos estudios que muestran la correlación positiva entre la IE y ciertas competencias sociales como: una mayor habilidad para identificar expresiones emocionales, un mayor apoyo social percibido y una mayor satisfacción con las relaciones establecidas en esa red social (Ciarrochi, Chan y Caputi, 2000). Al igual que una mayor puntuación en IE medida a través de (MEIS) se relaciona con una mayor empatía (Mayer, Caruso y Salovey, 1999). En esta misma línea, un estudio realizado por Extremera y Fernández-Berrocal (Extremera y Fernández-Berrocal, 2004b) postula como las habilidades de manejo emocional predicen mejores interacciones positivas y menores interacciones negativas, de modo que con una muestra de estudiantes universitarios evaluados mediante el MSCEIT, aquellos con niveles elevados de manejo emocional mostraban mayores niveles de intimidad y afecto hacia sus amigo, mayor implicación empática y una mayor toma de perspectiva así como menores niveles de malestar personal.

\subsection{Inteligencia emocional y conductas disruptivas}

En la actualidad, podemos encontrar una corriente internacional de investigación que está llevando a cabo estudios sobre el rol que desempeñan las competencias o, la falta de éstas, en los comportamientos disruptivos e incluso violentos en el ámbito educativo. Dichos estudios evidencian que una baja IE es un factor clave en la aparición de este tipo de conductas. Es esperable que el alumnado emocionalmente poco eficaz presente mayores niveles de impulsividad y peores habilidades interpersonales y sociales, lo cual favorece el desarrollo de diversos comportamientos antisociales. Las investigaciones desarrolladas por (Petrides, Frederickson y Furnham 2004) muestran como se relaciona la IE con el absentismo escolar, de modo que una deficiente competencia emocional se asocia con un mayor número de ausencias del centro no autorizadas. Por el contrario, el alumnado con un mayor nivel de habilidades afectivas presenta una mayor competencia en el uso de sus emociones como herramienta para obtener unas relaciones interpersonales adecuadas.

Numerosas investigaciones reflejan como la inestabilidad social del alumnado adolescente es predictiva de la conducta agresiva de éstos, de modo que los individuos más inestables cuentan con menores recursos para controlar su impulsividad, mientras que los más empáticos y que cuentan con una emocionalidad positiva y controlada, presentan mayores conductas prosociales (Maestre, Samper y Frias, 2002).

\subsection{Inteligencia emocional y consumo de sustancias}

En estudios realizados en EEUU (Trinidad y Johnson, 2002), se obtuvieron datos empíricos que constataban que una elevada IE medidos a través del (MEIS) se relaciona con un menor consumo de tabaco y alcohol en la adolescencia, evidenciando como factor de riesgo para el consumo de sustancias adictivas, el déficit emocional.

ntroc actidinc han anonntrado natronac mác acnorífionc onmo ac al actidin 
hallaron que unas bajas puntuaciones en los factores de percepción y asimilación, se relacionan con un mayor consumo de alcohol y drogas ilegales, un mayor número de peleas físicas y comportamientos adictivos y peores relaciones de amistad.

En España, en un estudio realizado entre 2007 y 2009 a 1467 adolescentes Malagueños, se obtuvo como resultado que los jóvenes con bajas competencias emocionales consumen más y son menos responsables con sus estudios; mientras que los que muestran eficacia emocional presentan mayor asertividad y el consumo de sustancias adictivas se centra en bebidas alcohólicas y tabaco. Se comprobó que las personas comprendidas entre los 13 y los 28 años que han probado algún tipo de sustancia adictiva y han mantenido el consumo en su vida cotidiana pierden capacidad de regular sus estados de ánimo y disminuyen la reacción de respuesta. (Ruiz-Aranda, Cabello, Martín-Salguero, Castillo, Extremera \& Fernández-Berrocal, 2010).

\subsection{Inteligencia emocional y rendimiento académico}

Una de las líneas de investigación que más interés ha generado en los últimos años en el ámbito de la IE ha sido el papel que ésta juega en el contexto educativo y, especialmente, en la influencia que tiene como determinante del éxito académico y la adaptación social de los estudiantes. Está claro que no podemos obviar la importancia de la cognición en el rendimiento del alumnado. No obstante, está demostrado empíricamente que ésta no es suficiente y que las competencias emocionales influencian tanto en el rendimiento académico como en el ámbito social.

La mayoría de los estudios realizados recientemente apoyan la relación existente entre IE y éxito académico, al igual que la relación entre IE y competencia social. (Fernández-Berrocal \& Extremera, 2006).

Las habilidades emocionales podrían contribuir a la adaptación social y académica de diversos modos; por un lado como facilitador del pensamiento, puesto que el trabajo cognitivo implica la capacidad de emplear y regular las emociones con objeto de favorecer la concentración, la impulsividad y manejo del estrés y, por tanto, producir un incremento en la motivación intrínseca del estudiante para realizar su trabajo escolar (Maestre, 2006); por otro lado, la IE podría influir sobre el desempeño académico confiriendo ventaja al alumnado emocionalmente competente en ciertas asignaturas relacionadas con el afecto, como puede ser el arte, la literatura, etc. (Petrides, Frederickson \& Furnhan, 2004)

Un estudio realizado con estudiantes universitarios australianos confirma que las altas puntuaciones en IE al principio del curso escolar se correlacionaban positivamente con la nota media del alumnado al finalizar el año. (Schutte, , Malouff, Hall, Haggerty, Cooper, Golden, y Dornheim, 1998). 
En definitiva, se puede afirmar que existe evidencia empírica del papel que juegan las emociones en el rendimiento del alumnado y como la IE influye de forma directa e indirecta en diversas áreas de funcionamiento, contribuyendo al bienestar y la adaptación del estudiante al sistema educativa. (Extremera \& Fernández-Berrocal, 2003) (Jiménez, 2009).

\section{METODOLOGÍA}

Con la implantación en las Universidades de la EEES y la remodelación en los planes de estudios, las competencias emocionales cobran si cabe, mayor importancia. De forma progresiva se están integrando diversas prácticas educativas en las que el peso de las relaciones sociales aumenta, como es: el trabajo en grupo y una mayor interacción entre el profesorado y los discentes.

En los aspectos teóricos en los que el trabajo individual y cognitivo del alumno tienen una mayor presencia, mientras que los aspectos más sociales quedan en un segundo plano, sería más fácil salir airoso con un trabajo responsable. Sin embargo, un alumno que presente déficit competencial en expresión, comprensión y/o regulación emocional, difícilmente obtendrá un alto rendimiento académico en las actividades de tipo más práctico en las que las relaciones sociales-emocionales adquieran protagonismo.

\subsection{Objetivos}

El objetivo principal es realizar un análisis en el que se correlacionen la variable "puntuaciones obtenidas en el TMMS-24" con la variable "calificaciones académicas". Ambas son cuantitativas y continuas. La primera puede adquirir valores desde el 24 al 120 en su escala total y 8-40 en cada una de sus tres sub-escalas (atención, claridad y regulación). La variable "calificaciones académicas" presenta dos modalidades: una es la calificación teórica y otra la calificación práctica. Ambas en una escala de 010.

Los objetivos específicos son:

a) Correlacionar la competencia autopercibida atención con las calificaciones académicas en la parte teórica y en la parte práctica;

b) Correlacionar la competencia autopercibida claridad con las calificaciones académicas tanto en la parte teórica y en la parte práctica;

c) Correlacionar la competencia autopercibida reparación con las calificaciones académicas en la parte teórica y en la parte práctica;

d) Realizar una comparación entre las evaluaciones de ambas partes en relación a las puntuaciones obtenidas en el TMMS-24. 


\subsection{Participantes}

La muestra está constituida por 147 estudiantes de $1^{\circ}$ curso de grado de la Universidad de La Rioja; 74 de grado de Educación Infantil y 73 de grado de Educación Primaria.

\subsection{Instrumentos}

En el presente estudio se administran las siguientes pruebas e instrumentos de medida:

a) TMMS-24. Se trata de una medida de autoinforme con una escala liker (15). Consta de 24 items que se dividen en tres sub-escalas: 1) Atención - Ser capaz de sentir y expresar los sentimientos de forma adecuada; 2) Claridad - Comprender bien los propios estados emocionales; 3) Regulación - Ser capaz de regular los estados emocionales correctamente.

b) Examen teórico. Se trata de una prueba escrita con cuestiones de tipología variada relacionadas con la materia proporcionada tanto en las clases teóricas como prácticas.

c) Rúbrica para las prácticas. Se trata de un informe que deben rellenar los propios alumnos sobre las prácticas de sus compañeros, en las que se puntúa: la relación con el grupo, la capacidad de comunicación e interacción al aula, la claridad en la exposición, etc.

\subsection{Procedimiento}

Para la realización de este estudio se realiza un análisis correlacional entre la variable puntuaciones obtenidas en el TMMS-24 sobre autopercepción en competencias emocionales y la variable calificaciones académicas en sus aspectos teóricos y prácticos. La metodología que se utiliza es principalmente cuantitativa.

El primero de los pasos es aplicar el TMMS-24 a la totalidad de los participantes. La valoración de esta herramienta se lleva a cabo teniendo en cuenta la baremación establecida por los autores, según las diferencias marcadas para hombres y mujeres. 
Tabla 1. Baremación del TMMS-24. Elaboración propia a partir de Fernández-Berrocal, Extremera \& Ramos, 2004.

\begin{tabular}{|c|c|c|c|c|c|c|}
\hline & & ATENCIÓN & & CLARIDAD & & REGULACIÓN \\
\hline $\mathrm{H}$ & \multirow{2}{*}{$z$} & $\begin{array}{c}<21 . \\
\text { Presta poca atención }\end{array}$ & \multirow[b]{2}{*}{ ت } & $\begin{array}{c}<25 \\
\text { Debe mejorar }\end{array}$ & \multirow{2}{*}{ 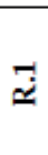 } & $<23$ Debe mejorar \\
\hline M & & $\begin{array}{c}<24 \\
\text { Presta poca atención }\end{array}$ & & $\begin{array}{c}<23 \\
\text { Debe mejorar }\end{array}$ & & $<23$ Debe mejorar \\
\hline $\mathrm{H}$ & \multirow{2}{*}{$\stackrel{3}{4}$} & $\begin{array}{c}22-32 \\
\text { Adecuada atención }\end{array}$ & \multirow{2}{*}{ 己ֶ } & $\begin{array}{c}26-35 \\
\text { Adecuada claridad }\end{array}$ & \multirow{2}{*}{ 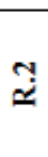 } & $\begin{array}{c}24-35 \\
\text { Adecuada reparación }\end{array}$ \\
\hline M & & $\begin{array}{c}25-35 \\
\text { Adecuada atención }\end{array}$ & & $\begin{array}{c}24-34 \\
\text { Adecuada claridad }\end{array}$ & & $\begin{array}{c}24-34 \\
\text { Adecuada reparación }\end{array}$ \\
\hline $\mathrm{H}$ & \multirow{2}{*}{$\stackrel{3}{4}$} & $\begin{array}{c}>33 \text { Presta demasiada } \\
\text { atención }\end{array}$ & \multirow{2}{*}{ ? } & $\begin{array}{c}>36 \\
\text { Excelente claridad }\end{array}$ & \multirow{2}{*}{$\stackrel{m}{\simeq}$} & $\begin{array}{c}>>36 \\
\text { Excelente reparación }\end{array}$ \\
\hline M & & $\begin{array}{c}>36 \text { Presta demasiada } \\
\text { atención }\end{array}$ & & $\begin{array}{c}>35 \\
\text { Excelente claridad }\end{array}$ & & $\begin{array}{c}>35 \\
\text { Excelente reparación }\end{array}$ \\
\hline
\end{tabular}

Una vez obtenidas las puntuaciones se distribuye a los alumnos en 9 grupos: 3 para la sub-escala atención; 3 en la sub-escala claridad y 3 en la sub-escala regulación. Cada alumno formará parte de 3 grupos, uno por sub-escala.

El segundo momento en el estudio, se refiere a la evaluación de la parte teórica realizada a través de una prueba escrita con cuestiones sobre los aspectos teóricos expuestos en el aula. La valoración por parte del docente se lleva a cabo con los criterios de evaluación presentes en las guías de cada asignatura.

A lo largo del curso se desarrolla la evaluación de la parte práctica a través de una rúbrica que debe cumplimentar el resto de los alumnos, en la que se puntúa a cada sujeto en una escala 0-10. Posteriormente se realiza la media numérica entre el total de las rúbricas. Esta media va a suponer la calificación de la parte práctica de cada participante.

\subsection{Análisis de datos}

El análisis estadístico de las diferentes variables se ha realizado a tres niveles. Un primer nivel de carácter descriptivo de toda la muestra y dividida por los grupos establecidos según las puntuaciones obtenidas en el TMMS-24 en cada una de las sub-escalas.

El segundo nivel, también de carácter descriptivo, se trata de la comparación de los promedios en las calificaciones de teoría y práctica, según los cuartiles establecidos con las puntuaciones obtenidas en el TMMS-24.

El último nivel se centra en el análisis correlacional de las variables, aplicando la prueba de Pearson emparejando tanto la puntuación total como la puntuación de las tres sub-escalas del TMMS-24 con las calificaciones obtenidas en la parte teórica y 


\section{ANÁLISIS Y DISCUSIÓN}

En la tabla 2 se detallan los principales datos estadísticos descriptivos de la muestra, segmentada por grupos según las puntuaciones obtenidas en las diferentes subescalas, de modo que A1= Debe mejorar, presta poca atención a sus emociones; $\mathrm{A} 2=$ Atención adecuada; $\mathrm{A} 3=$ Debe mejorar, presta demasiada atención a sus emociones; $\mathrm{C} 1=$ Debe mejorar su claridad; $\mathrm{C} 2=$ Adecuada claridad; $\mathrm{C} 3=$ Excelente claridad; $\mathrm{R} 1=$ Debe mejorar su reparación; R2= Adecuada reparación; R3= Excelente reparación.

Se puede observar como las medias de las calificaciones obtenidas son mayores en la parte práctica en todos los casos, exceptuando el caso de A3 donde existe un leve incremento en la parte teórica.

Tabla 2. Principales datos estadísticos del análisis

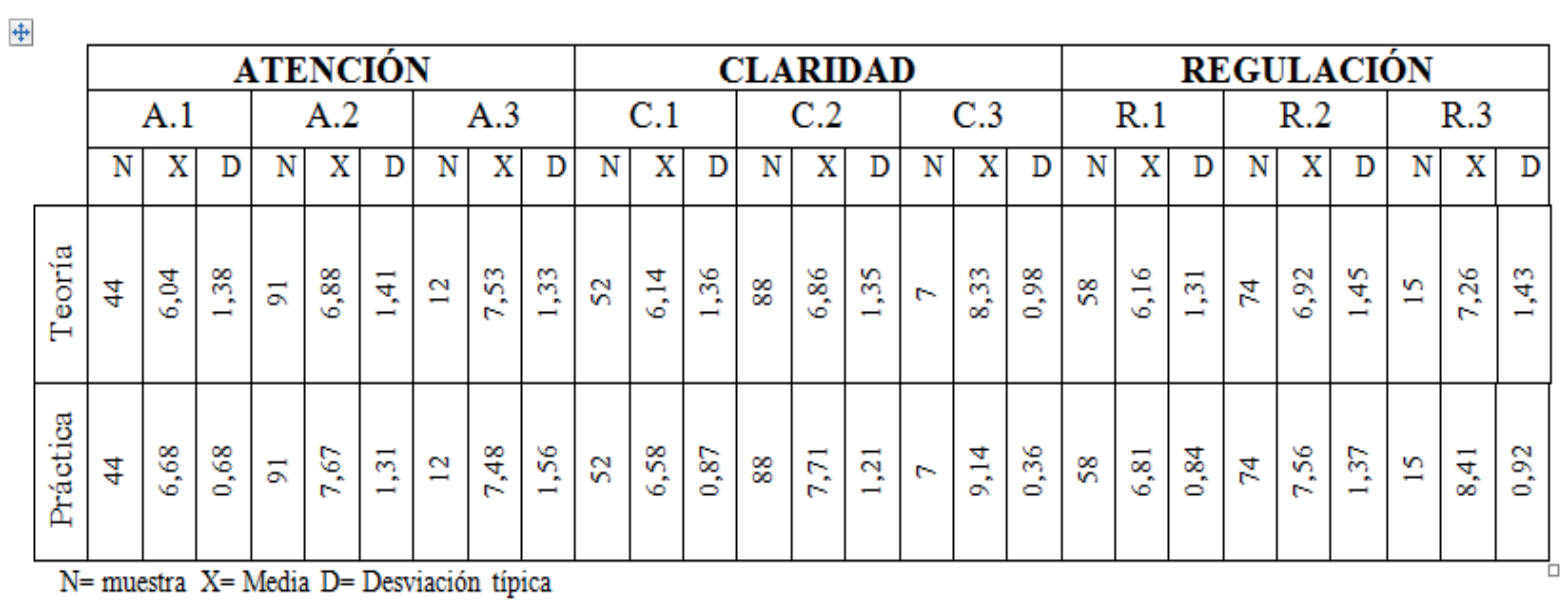

La figura 1 muestra las puntuaciones obtenidas en las partes teórica y práctica en las diferentes sub-escalas de la herramienta de autoinforme, según las agrupaciones realizadas. Se puede observar como se produce un incremento en las calificaciones a medida que aumentan las puntuaciones en IE, valorada según el TMMS-24. Este hecho ocurre en todas las sub-escalas de la herramienta a excepción de la A3 en el que se aprecia un leve descenso. Se debe tener un cuenta que esta sub-escala presenta la característica de que una puntuación elevada se corresponde con una excesiva atención a las emociones lo que no es adecuado. 

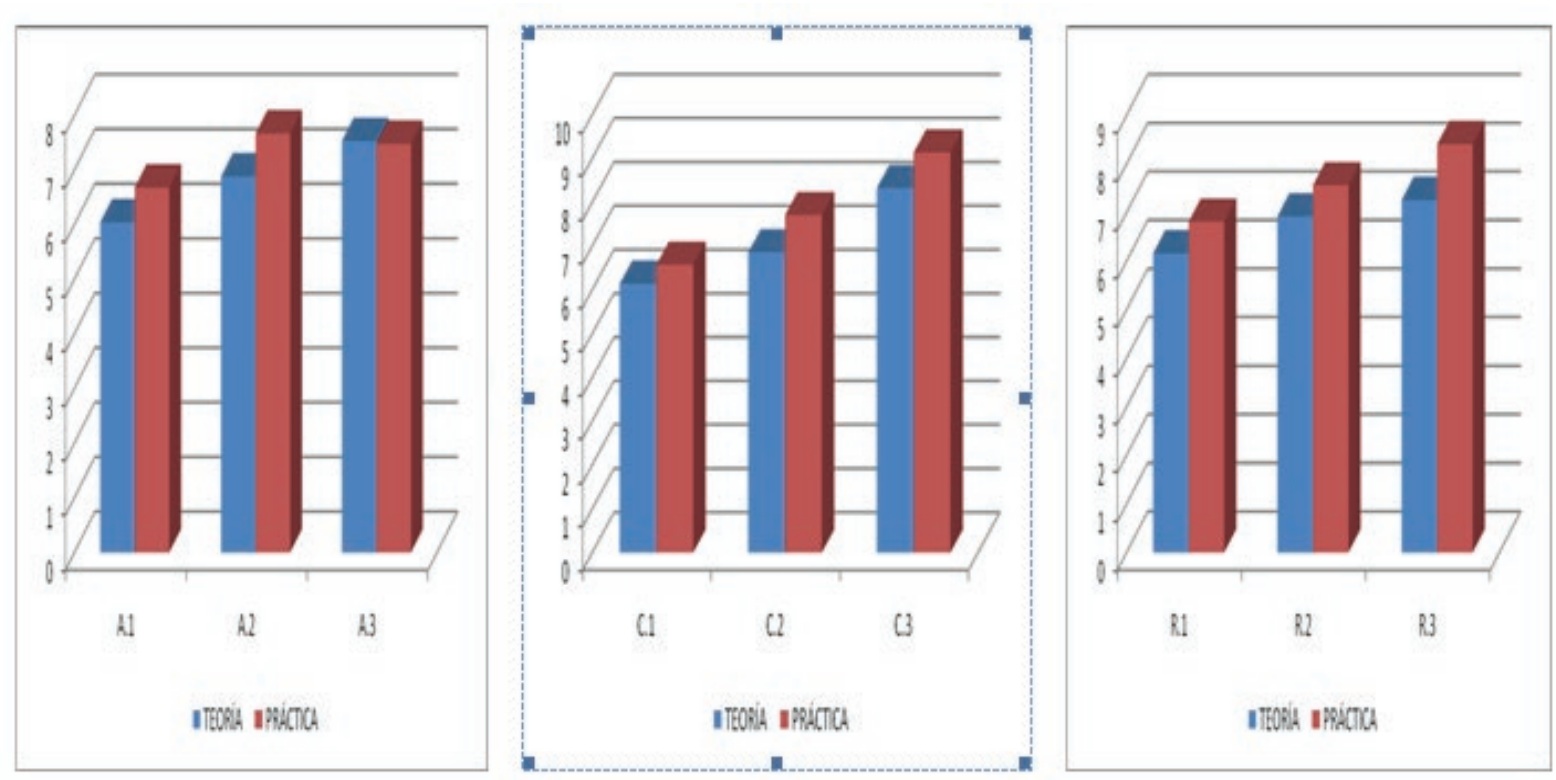

Figura 1. Calificaciones obtenidas según las diferencias sub-escalas

Las tabla 3 y la figura 2 muestran los promedios obtenidos por los alumnos, agrupados en cuartiles según las puntuaciones totales obtenidas en el TMMS-24, en las calificaciones tanto de la parte teórica como práctica.

Tabla 3. Resultados en teoría y práctica distribuidos por cuartiles

\begin{tabular}{c|ccc}
\hline & $1^{\circ}$ cuar. & $2^{\circ}$ cuar. & $3^{\circ}$ cuar. \\
\hline Teoría & $\mathbf{5 , 5 3}$ & $\mathbf{6 , 5 8}$ & $\mathbf{8 , 1 1}$ \\
Práctica & $\mathbf{6 , 5 0}$ & $\mathbf{6 , 9 6}$ & $\mathbf{9 , 0 6}$ \\
\hline
\end{tabular}

Se puede apreciar como ambas sufren un incremento a medida que aumenta la variable "puntuaciones obtenidas en el TMMS-24" 


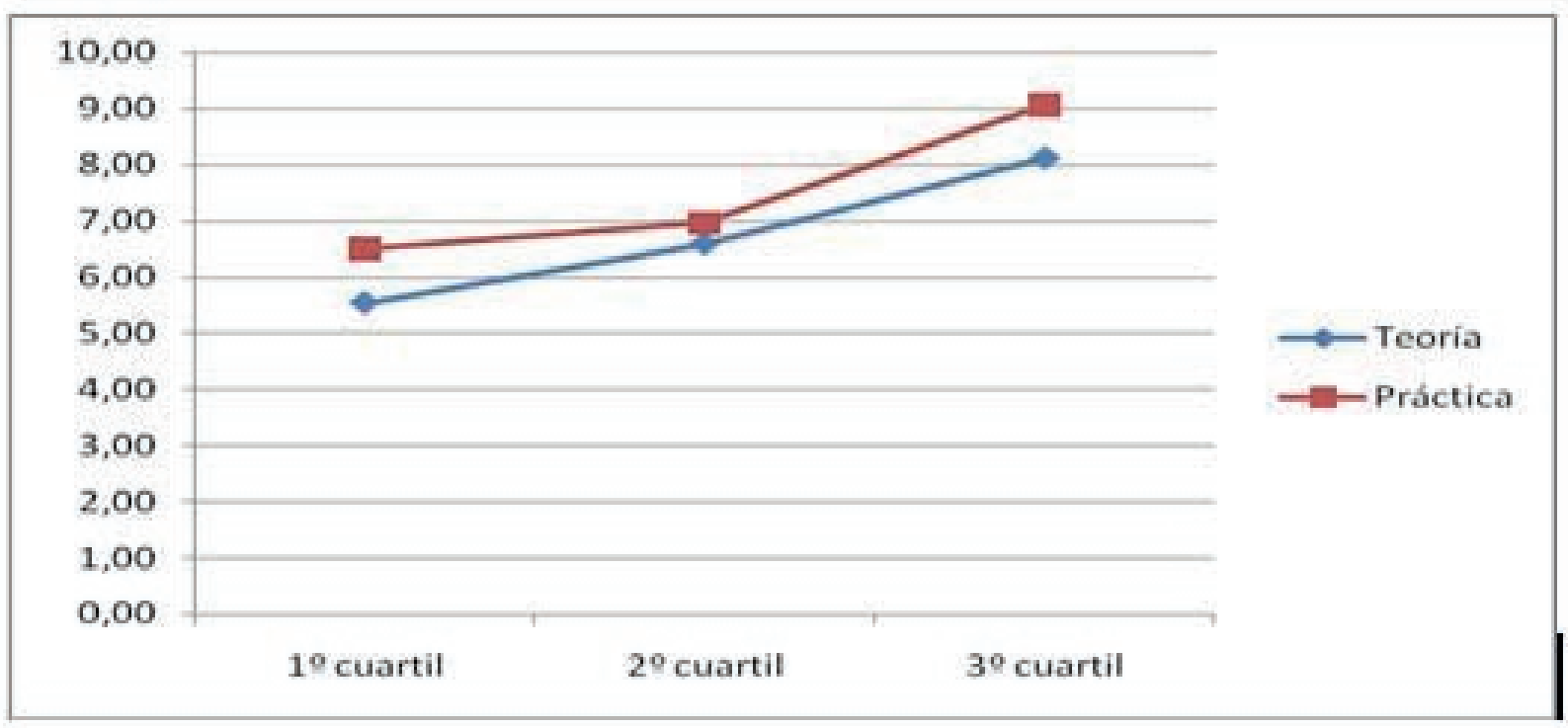

Figura 2. Calificaciones en teoría y práctica distribuida según cuartiles.

A continuación se realiza el cálculo de las correlaciones existentes tanto en la puntuación total como en las tres sub-escalas del TMMS-24 y, las calificaciones obtenidas en la parte práctica y en la teórica. Para ello se utiliza el estadístico coeficiente de correlación de Pearson (rxy).

Tabla 4. Matriz de correlaciones entre las variables del estudio

\begin{tabular}{lcccc}
\hline & Punt. total & Atención & Claridad & Regulación \\
\hline Cal. Teórica &, 576 &, 359 &, 405 &, 371 \\
Cal. Práctica &, 751 &, 429 &, 588 &, 466 \\
\hline
\end{tabular}

Los valores de las correlaciones oscilan desde el valor más alto $\mathrm{r}=0,751$ entre la puntuación total del TMMS-24 y la calificación de la parte práctica, hasta el valor más bajo $r=0,359$ entre la sub-escala atención y la calificación teórica. Todos los coeficientes son positivos, por lo que se determina que a medida que existe un incremento en la IE se incrementan las calificaciones académicas. La correlación es más fuerte en la parte práctica.

En la franja de correlaciones estimadas como moderada se encuentran la sub-escala atención con ambas calificaciones. La sub-escala claridad con la calificación teórica y la sub-escala regulación con la puntuación en las partes teórica y práctica. En intervalo de correlación moderada alta se encuentra la correlación entre puntuación total en la escala de autoinforme y la calificación teórica, también se sitúa en este tramo la sub-escala claridad con la calificaciones en la práctica. La correlación entre la puntuación total y la calificación práctica se sitúa entre moderada-alta y alta. 
Como anteriormente se ha venido apuntando existe un importante número de investigaciones que relacionan las competencias emocionales con el rendimiento escolar. Los resultados obtenidos en este estudio vinculan las puntuaciones de la escala de autoinforme TMMS-24 con las calificaciones en el alumnado universitario de modo que: a mayor puntuación en la escala, mejores calificaciones tanto en la parte teórica como práctica. En este sentido se encuentran los trabajos de Maestre y col, 2006 que postulan que la eficacia en competencias emocionales podrán contribuir a la adaptación social y académica actuando como facilitador del pensamiento, puesto que el trabajo cognitivo implica la capacidad de emplear y regular las emociones con objeto de favorecer la concentración, la impulsividad y el manejo del estrés $\mathrm{y}$, por tanto, producir un incremento en la motivación intrínseca del estudiante. Los datos obtenidos que correlacionan tanto la puntuación total de la escala, como la de las tres sub-escalas que la forman, corroboran este hecho, apreciándose un aumento en las calificaciones a favor del alumnado con mayores competencias emocionales.

Si esta correlación se produce en la parte teórica, podemos apreciar una acentuación en la parte práctica. Esto puede ser debido a que la metodología de este apartado, además de un importante componente cognitivo, conlleva el trabajo en grupo, cobrando un mayor peso habilidades como la empatía, el saber escuchar, la identificación de las emociones del otro, la comunicación asertiva, la regulación y control de impulsos, etc.

En este sentido se dirigen los trabajos de Ciarrochi, Chan y Caputi, 2000, mostrando una relación positiva entre la IE y una mayor habilidad para identificar expresiones emocionales, un mayor apoyo social percibido y una mayor satisfacción con las relaciones interpersonales establecidas. Otra investigación en esta misma línea es la desarrollada por Extremera y Fernández-Berrocal (Extremera \& FernándezBerrocal 2004b) indicando como las habilidades de manejo emocional predicen mejores interacciones positivas y menores interacciones negativas, muestran mayores niveles de intimidad y afecto hacía sus amigos y una mayor empatía.

Este estudio pretende, por un lado ser una discreta contribución a las investigaciones que muestra las correlaciones positivas entre competencias emocionales y rendimiento académico. Se debe tener presente que las aulas son un contexto de variadas y continuadas relaciones interpersonales $y$, que dichas relaciones sociales están mediatizadas por unas eficaces competencias emocionales. Por otro lado, persigue la finalidad de evidenciar, de un modo indirecto, los beneficios de la inclusión de la Educación Emocional en las diferentes etapas educativas. Las universidades no pueden obviar el trabajo emocional en sus aulas.

Se debe introducir la educación socio-afectiva con objeto de formar futuros profesionales competentes en trabajo en equipo y en relaciones interpersonales, tal y

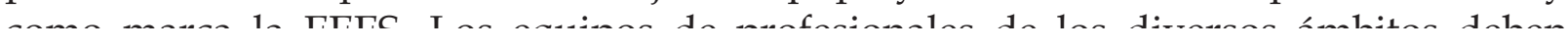


propósitos, propuestas, planes, etc. Para que un equipo sea eficaz, los miembros que la constituyen deben poseer habilidades socio-emocionales como: asertividad, capacidad para comunicar de forma efectiva, autoestima, habilidad para manejar las propias emociones, identificar las de los demás y respetarlas, ser empáticos, controlar las emociones negativas y gestionarlas correctamente, etc.

\section{REFERENCIAS}

Bar-on, R. \& Handley, R. (1997). The Emotional Quotient 360 (EQ-360): A multirater assessment for emotional intelligence. Toronto, Canada: Multi-Health Systems.

Boyachis, R. \& Burckel, N. (1999). Psychometric properties of the ECI. Boston: MA. Hay/Mcber Group.

Brackett, M. \& Mayer, J. (2003). Emotional intelligence and its relation to everyday behavior. Personality and Individual Differences, 28(3): 539-561.

Ciarrochi, J. V., Chan, A. \& Caputi, P. (2000). A critical evaluation of the emotional intelligence construct. Personality and Individual differences, 28(3): 561-570.

Extremera, N. \& Fernández-Berrocal, P. (2003). La inteligencia emocional en el contexto educativo: Hallazgos científicos de sus efectos en el aula. Revista de Educación, 332 (2003): 97-116.

Extremera, N. \& Fernández-Berrocal, P. (2004a). ¿Cómo se mide la inteligencia emocinal? En Inteligencia emocinal. La relación entre pensamienos y sentimientos en la vida cotidiana. Biblioteca Nueva. Madrid.

Extremera, N. \& Fernández-Berrocal, P. (2004b). Inteligencia emocional, calidad de las relaciones interpersonales y empatía en estudiantes universitarios. Clínica y Salud, 15(2): 117-137.

Fernández-Berrocal, P., Alcaide, R. \& Ramos, N. (1999). The influence of emotional intelligence on the emotional adjustment in highschool students. Bulletin so Kharkov State University, 439(1-2): 119-123.

Fernández-Berrocal, P., Alcaide, R., Extremera, N. \& Pizarro, D. (2006): The role of emotional intelligence in anxiety and depression among adolescents. Individual Differences Research, 4: 16-27.

Fernández- Berrocal, P. \& Extremera, N. (2005): La inteligencia emocional y la educación de las emociones desde el modelo de Mayer y Salovey. Revista Interuniversitaria de Formación del Profesorado, 19 (3): 63-93. 
the Spanish Modified Version of the Trait Mete-Mood Scale. Psychological Reports. 94: 751-755.

Fernández-Berrocal, P. \& Extremera, N. (2006). Emotional Intelligence: A theoretical and empirical review of its first 15 years of history. Psicothema, 18: 7-18.

Jiménez, I. (2009). Una aproximación psicosocial a la relación entre inteligencia emocional y rendimiento académico. Jaén: Universidad de Jaén.

Maestre, J. M., Güil, R., Lopes, P., Salovey, P. \& Gil-Olarte, P. (2006). Emotional Intelligence and Social and academic adaptation to school. Psicothema, 18: 112-117.

Maestre, M. V., Samper, P. \& Frias, M. D. (2002). Procesos cognitivos y emocionales predictores de la conducta prosocial y agresiva: La empatía como factor modular. Psicothema, 14(2): 227-232.

Mayer, J., Caruso, D. R \& Salovey, P. (1999). Emotional intelligence meets traditional standards for an intelligence. Intelligence 27: 267-298.

Mayer, J., Caruso, D. R \& Salovey, P. (2002). Mayer- Salovey- Caruso Emocional Intelligence Test (MSCEIT) Item Booklet. Toronto, Canada: MHS Publishers.

Petrides, K. V., Frederickson, N. \& Furnhan, A. (2004). The role of trait emotional intelligence in adademic performance and deviant behavior at school. Personality and individual differences, 36(2): 277-293.

Petrides, K. V. \& Furnhan, A. (2001). Trait emotional intelligence: Psychometric investigation with reference to established trait taxonomies. European Journal of Personality, 15: 425- 448.

Ruiz-Aranda, D., Cabello, R., Martín-Salguero, J., Castillo, R., Extremera, N. \& Fernández-Berrocal, P. (2010). Los adolescentes Malagueños ante las drogas: la influencia de la Inteligencia Emocional. Consultado el 03 de octubre de 2010, Disponible en:

http:/ / www.uma.es/contenido.php?idm=29\&clase=p\&id=2068\&tipo=n.

Salovey, P., Mayer, J., Golman, S., Turvey, C. \& Palfai, T. P. (1995). Emotional attention, clarity, and repair: Exploring emotional intelligence using te Trait MetaMood Scale En Emotion, disclosure, and Elath. Washington, DC: J.W. Pennebaker.

Salovey, P., Strou, L., Woolery, A. \& EPEL, E. S (2002). Perceptived Emotional Intelligence: Conceptualization and measuremet En Blackwell handbook of social 
Schutte, N., Malouff, J. M, Hall, L. E, Haggerty, D. J, Cooper, J. T, Golden, Ch. \& Dornheim, L. (1998). Development and validation of measure of emotional intelligence. Personality an individual Difference, 25: 167-177.

Trentacosta, Ch. \& Izard, C. E. (2007). Kindergarten children's emotion competence as a predictor of their competence in first grade. Emotional, 7: 77-88.

Trinidad, D. R. \& Johnson, A. (2002). The association between emotional intelligence and early adolescent et tabacoo and alcohol use. Personality and Individual Differences, 32(1): 95-105.

\section{Iratxe Suberviola-Ovejas}

Licenciada en Psicopedagogía, con el Diploma de Estudios Avanzados en la modalidad de Didáctica y Organización Escolar. Profesora del área de Teoría e Historia de la Educación en la Universidad de la Rioja. Actualmente estoy realizando la tesis doctoral con temática relacionada con la educación y las competencias emocionales. Las líneas de investigación desarrolladas son: 1) inteligencia y educación emocional; 2) género y coeducación. Autora de artículos y capítulos de libro relacionados con estas materias. Miembro del G.I de la Universidad de la Rioja: Igualdad y Género. Investigadora del Instituto de Estudios Riojanos. (IER). 